\title{
Sistemas adhesivos autograbadores en esmalte: ventajas e inconvenientes
}

\author{
Gomes Moreira M.A*
}

\section{RESUMEN}

Se mide la resistencia adhesiva de cinco sistemas adhesivos autograbadores y se compara con dos monocomponentes, en esmalte bovino, utilizando cuatro protocolos de aplicación diferentes (según fabricante, con ácido, dos tiempos y dos capas). Asimismo se evalúan con MEB los patrones de grabado ácido de estos sistemas adhesivos, muy heterogéneos en los autograbadores. Todos los sistemas autograbadores presentan valores aceptables de resistencia adhesiva.

Palabras clave: Adhesivos autograbadores, esmalte y patrón de grabado ácido.

\begin{abstract}
The bond strength to bovine enamel of five self-etching adhesive systems was measured comparing to two total etch adhesive systems and using different application modes (following manufacturers' instructions, with a previous acid etching, duplicating priming time and doubling the number of adhesives' layers). The etching patterns were analized with scanning electron microscopy showing the self-etching adhesives different aggressiveness. All self-etching systems showed reliable bond strength values.
\end{abstract}

Key words: Self-etching adhesives, enamel and etching pattern.

Aceptado para publicación: Octubre 2003

* Becario de Investigación. Facultad de Odontología. Universidad de Granada.

Gomes Moreira M.A. Sistemas adhesivos autograbadores en esmalte: ventajas e inconvenientes. $A v$. Odontoestomatol 2004; 20-4: 193-198.

\section{INTRODUCCIÓN}

La demanda constante y creciente, ocurrida durante los últimos años, de tratamientos estéticos, restauradores o no, por parte de nuestros pacientes ha estimulado la aparición de nuevos materiales e instrumental, con el fin de facilitar y simplificar el trabajo de los profesionales de la Odontología. De este modo, cada año los fabricantes nos presentan innumerables novedades, principalmente materiales para ser utilizados en procedimientos estéticos como son el blanqueamiento dental y las restauraciones adhesivas.

Los sistemas adhesivos son de los materiales odontológicos más estudiados e interesantes para los investigadores, pues constituyen un grupo del que dependen la mayoría de los procedimientos restauradores relacionados con la estética dental. Los estudios sobre adhesión al esmalte, dentina y cemento constituyen una gran parte de las investigaciones realizadas en el campo de la Odontología, y las principales variables que se evalúan son la microfiltración y la resistencia adhesiva producidas en los distintos substratos dentarios utilizando todo tipo de materiales restauradores.

La técnica del acondicionamiento con ácido fosfórico propuesta por Buonocore en 1955 revolucionó la odontología restauradora e hizo que se pudieran obtener mejores resultados durante los procedi- 
mientos adhesivos. Esta técnica se basa en el tratamiento ácido del esmalte, transformando su superficie lisa y suave en una superficie acentuadamente irregular y con mayor energía superficial. Aunque el acondicionamiento del esmalte con ácido fosfórico previo a la aplicación del sistema adhesivo es aún la técnica más recomendada en el esmalte, algunos de los sistemas adhesivos más modernos, denominados autograbadores, se consideran por algunos autores alternativas eficaces para asegurar una adhesión duradera y un buen sellado marginal en las restauraciones de composite.

Los sistemas adhesivos autograbadores son sistemas que presentan los agentes grabadores e imprimadores juntos y que disuelven el barrido dentinario y lo incorporan en el proceso adhesivo además de demineralizar parcialmente la subsuperficie de la dentina. Inicialmente estos sistemas estaban indicados solamente para procedimientos adhesivos en dentina, sin embargo, algunos de los sistemas autograbadores actuales presentan patrones de grabado del esmalte similares a los producidos por el acondicionamiento con ácido fosfórico. Además, los valores de fuerza de adhesión son comparables o incluso superiores a los obtenidos con la técnica convencional de grabado ácido.

\section{VENTAJAS E INCONVENIENTES DE LOS SISTEMAS AUTOGRABADORES}

Las principales ventajas de los sistemas autograbadores son :

- La desmineralización e infiltración de la dentina ocurren simultáneamente;

- Durante el procedimiento adhesivo no hay que lavar tras el grabado, por eso se considera una técnica más rápida;

- No son tan sensibles a las diversas condiciones de humedad de la dentina;

- Son poco sensibles a la técnica;

- Se pueden utilizar como materiales desensibilizantes;

- Su aplicación es higiénica;

- Presentan una composición consistente y estable.
Los principales inconvenientes son:

- Los estudios a largo plazo son todavía insuficientes;

- Aún se requieren más pruebas clínicas referentes a la adhesión al esmalte.

\section{OBJETIVOS}

Los objetivos principales de este estudio fueron:

1) Medir la resistencia adhesiva de cinco sistemas adhesivos autograbadores en esmalte bovino, utilizando cuatro protocolos de aplicación diferentes, mediante un ensayo de microtensión. Así como comparar los resultados obtenidos con los conseguidos por dos sistemas adhesivos monocomponentes utilizados con previo grabado ácido del esmalte.

2) Evaluar a través de microscopia electrónica de barrido (MEB) los patrones de grabado ácido producidos por algunos sistemas adhesivos autograbadores en esmalte bovino y compararlos con el patrón obtenido con el ácido fosfórico.

\section{MATERIAL Y MÉTODO}

\section{- Evaluación de la resistencia adhesiva}

Para la realización del presente estudio decidimos utilizar incisivos bovinos sanos. Estos dientes se consideran buenos sustitutos de los dientes humanos cuando se llevan a cabo estudios de adhesión, puesto que presentan menos variaciones en su estructura y su manipulación es más sencilla debido a su mayor tamaño. Además, su microestructura y la orientación de los cristales de hidroxiapatita son similares a las del humano.

Los sistemas adhesivos autograbadores utilizados fueron Clearfil SE Bond (Kuraray Co.Ltd), Prompt-LPop (ESPE), NRC-Non-Rinse Conditoner + Prime $\mathcal{E}$ Bond NT (Dentsply De Trey), Resulcin Aquaprime + Monobond (Merz Dental) y Etch \& Prime 3.0 (Degussa AG). Los sistemas adhesivos monocomponentes utilizados fueron Prime $\&$ Bond NT (Dentsply De Trey) y Scotchbond 1 (3M). La resina compuesta empleada fue Tetric Ceram (Ivoclar/Vivadent). 
Para preparar los especimenes seguimos la siguiente metodología: Se pulieron las superficies vestibulares de los dientes utilizando discos de $\mathrm{SiC}$ de 220 y 500 grit en una pulidora mecánica (EXAKTApparatebau, Otto Herrman, Norderstedt, Alemania) hasta obtener una superficie plana de esmalte. Se eliminaron las raíces y se rellenó la cámara pulpar con resina compuesta. Para aumentar la longitud de las barritas en el tercio incisal y así poder medirlas en el aparato para pruebas de microtensión se eliminó la capa superficial de esmalte aprismático y se adhirió una capa de composite en la superficie palatina.

Las superficies vestibulares fueron tratadas con los adhesivos anteriormente expuestos y se aplicaron de cuatro modos diferentes: 1 . Según las indicaciones de cada fabricante (según fabricante); 2. Realizando el grabado con ácido fosfórico del esmalte previamente (con ácido); 3. Dejando actuar el adhesivo el doble de tiempo del recomendado por los fabricantes antes de la fotopolimerización (2 tiempos); 4.Aplicando el doble de capas de adhesivo que las recomendadas por cada fabricante ( 2 capas).

Se construyó una capa final de composite Tetric Ceram (Ivoclar/Vivadent) de aproximadamente $6 \mathrm{~mm}$, colocando incrementos de aproximadamente $2 \mathrm{~mm}$ y fotopolimerizando durante 40 s cada incremento. Los especímenes fueron conservados a $37^{\circ} \mathrm{C}$ durante $24 \mathrm{~h}$ y posteriormente seccionados en ambos ejes "x" e "y" para obtener barritas de aproximadamente $1 \mathrm{~mm}^{2}$ de sección. Los especímenes fueron sometidos a tensión en una máquina de tracción universal Instron (Instron Limited Corporation, 4411, Barcelona, España) con una velocidad de travesaño de $0,5 \mathrm{~mm} / \mathrm{min}$. Tras determinar el área de cada barrita después de su fractura, los datos fueron calculados en MPa. Los datos obtenidos se analizaron mediante un ANOVA de dos vías incluyendo interacciones de segundo orden y comparaciones múltiples con el test de Student-Newman-Keuls $(p<0,05)$ (SPSS /PC + V.4.0. 1990).

\section{- Evaluación de los patrones de grabado}

Se realizó el mismo protocolo de preparación de los especimenes y se aplicaron los diferentes sistemas autograbadores sometidos a estudio en las superfi- cies vestibulares pulidas, en este caso, siguiendo las recomendaciones de los fabricante, pero sin polimerizarlos. A continuación, las superficies grabadas se introdujeron en baños con concentraciones crecientes de etanol (50, 60, 70, 80, 90 y 96\%), con un tiempo de inmersión de un minuto en cada solución. Más tarde se incluyeron en etanol puro durante 1 minuto en ultrasonidos con el fin de disolver completamente cualquier residuo de primer o adhesivo. Los especímenes, una vez procesados, se cementaron en un portamuestras de aluminio para microscopio electrónico de barrido con un pegamento con carbón. A continuación se cubrieron con oro utilizando una unidad de cobertura Polaron E-5000 (Polaron Equipment Ltd. Watford, Inglaterra). Los especímenes fueron observados en un microscopio electrónico de barrido ZEISS DSM-950 (Carl Zeiss, Alemania).

\section{RESULTADOS}

Cuando se analizaron los resultados de resistencia adhesiva de los sistemas adhesivos estudiados, según el modo de aplicación utilizado, se observó que:

1. SEGÚN FABRICANTE: No hubo diferencias estadísticamente significativas entre los valores de resistencia adhesiva de los diferentes sistemas adhesivos.

2. CON ÁCIDO: El sistema Etch \& Prime 3.0 presentó valores de resistencia adhesiva más altos que los valores presentados por los demás adhesivos.

3. 2 TIEMPOS: El sistema Etch $\&$ Prime 3.0 presentó valores de resistencia adhesiva estadísticamente más altos que los valores presentados por los demás adhesivos. El sistema NRC + Prime $\mathcal{E}$ Bond NT presentó valores estadísticamente más bajos que el resto.

4. 2 CAPAS: Los sistemas Etch \& Prime 3.0, Scotchbond 1 y Prompt-L-Pop presentaron valores de resistencia adhesiva estadísticamente más altos que los valores presentados por los sistemas Prime $\varepsilon$ Bond NT, NRC + Prime $\varepsilon$ bond NT, Clearfil SE Bond y Resulcin. 
Evaluando los resultados de cada sistema adhesivo en las diversas formas de aplicación, se observó que:

5. PRIME \& BOND NT: Los valores de resistencia adhesiva de este adhesivo cuando se aplicó en un número doble de capas ( 2 capas) fueron estadísticamente inferiores a los obtenidos cuando se empleó según fabricante (con ácido) y con un tiempo de aplicación doble (2 tiempos). Se supone que por ser un sistema que contiene nanorelleno inorgánico, ya presenta una capa con un grosor ideal para proporcionar una buena resistencia adhesiva.

6. SCOTCHBOND 1, PROMPT-L-POP, CLEARFIL SE BOND Y NRC + PRIME \& BOND NT: Los valores de resistencia adhesiva de cada uno de estos sistemas adhesivos fueron estadísticamente similares independientemente del modo de aplicación.

7. ETCH \& PRIME 3.0: Los valores de resistencia adhesiva de este sistema adhesivo, utilizado según las indicaciones del fabricante, fueron estadísticamente más bajos que cuando se empleó siguiendo otros modos de aplicación. El hecho de ser un sistema adhesivo que presenta un patrón poco agresivo de grabado ácido puede justificar tal resultado.

8. RESULCIN: Con este sistema adhesivo se obtuvieron valores estadísticamente diferentes entre las

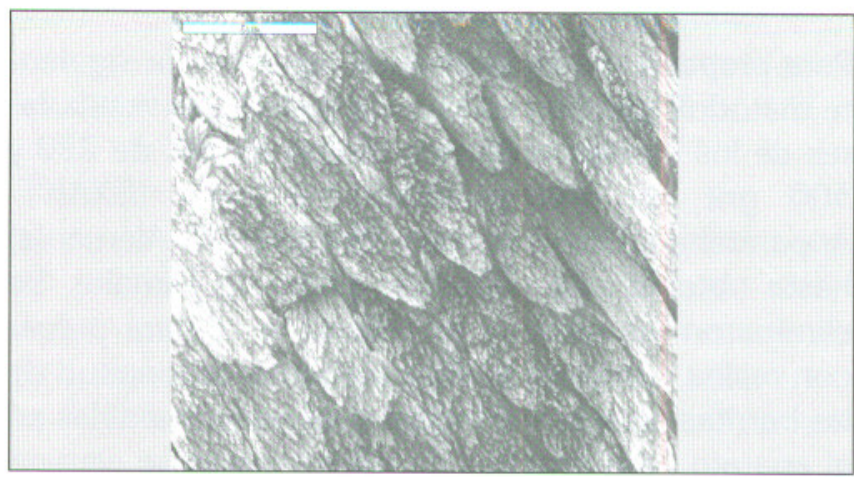

Fig. 1. Patrón de grabado del ácido Ortofosfórico a 37\% utilizado durante 30 s.

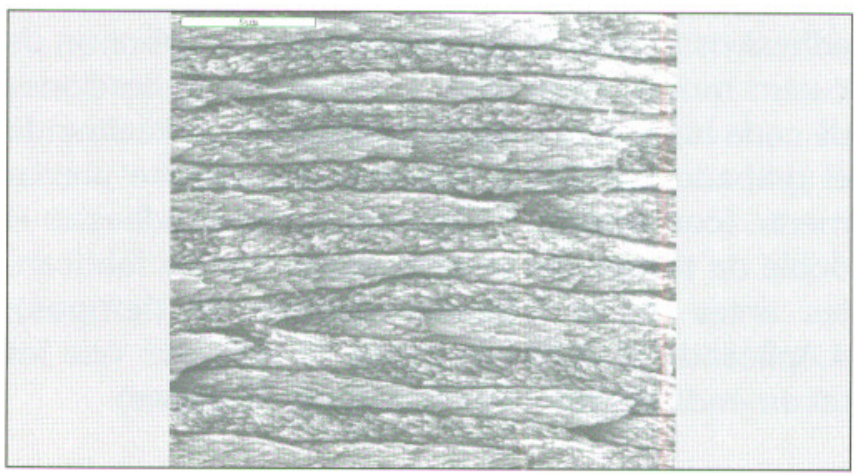

Fig. 2. Patrón de grabado del Prompt-L-Pop utilizado siguiendo las instrucciones del fabricante.

formas de manipulación de 2 tiempos (valores más altos) y de 2 capas ( valores más bajos).

Los valores de resistencia adhesiva de los sistemas

\section{TABLA 1.- VALORES DE RESISTENCIA ADHESIVA EN MPa (MEDIA Y DESVIACIÓN ESTÁNDAR) DE CADA UNO DE LOS SISTEMAS ADHESIVOS EVALUADOS, SEGÚN CADA VARIABLE DE APLICACIÓN}

\begin{tabular}{|l|c|c|c|c|}
\hline MANIPCILACIÓN & $\begin{array}{c}\text { S. FAB } \\
x(D E)\end{array}$ & $\begin{array}{c}\text { C/ ÁCIDO } \\
x(D E)\end{array}$ & $\begin{array}{c}2 T \\
x(D E)\end{array}$ & $\begin{array}{c}2 C \\
\text { S. ADHESIVOS }\end{array}$ \\
\hline Prime \& Bond NT & $26,92(5,48)$ & - & $29,89(6,20)$ & $18,01(4,88)$ \\
\hline Scotchbond 1 & $29,30(9,84)$ & - & $27,90(8,82)$ & $30,84(9,62)$ \\
\hline Prompt-L-Pop & $28,32(7,40)$ & $29,51(8,82)$ & $31,91(6,84)$ & $25,35(4,76)$ \\
\hline Clearfil SE Bond & $22,10(10,64)$ & $21,39(6,42)$ & $23,27(6,98)$ & $15,86(5,50)$ \\
\hline Etch E Prime & $28,90(4,90)$ & $34,41(6,68)$ & $39,61(6,34)$ & $34,45(6,12)$ \\
\hline Resulcin & $26,06(7,36)$ & $24,55(5,58)$ & $28,43(6,74)$ & $19,17(3,00)$ \\
\hline NRC + Prime E Bond NT & $24,53(10,36)$ & $21,64(6,08)$ & $17,84(6,32)$ & $20,18(9,62)$ \\
\hline
\end{tabular}


adhesivos utilizados según cada forma de aplicación están reflejados en la tabla.1

En cuanto al análisis de las superficies con microscopia electrónica de barrido, cabe destacar que se observaron patrones de grabado muy heterogéneos, desde patrones agresivos como el obtenido con el ácido fosfórico (Fig.1), tal y como ocurrió con Prompt-L-Pop (Fig.2) y Resulcin, hasta patrones poco agresivos como el del Etch $E$ Prime o irregulares como el del Clearfil SE Bond.

\section{CONCLUSIONES}

1. Aunque algunos sistemas adhesivos autograbadores presenten patrones de grabado ácido menos agresivos comparados con el patrón obtenido por el acondicionamiento convencional con ácido fosfórico, todos presentan valores aceptables de resistencia adhesiva.

2. Todos los sistemas adhesivos autograbadores utilizados se han estudiado a corto plazo.

3. Todos estos sistemas adhesivos autograbadores se pueden utilizar en el esmalte siguiendo las instrucciones de los fabricantes.

\section{AGRADECIMIENTOS}

Este estudio ha sido financiado por el proyecto CICYT - MAT \# 2843-CO2 dirigido por el Dr. Manuel Toledano Pérez (Prof. Titular del Departamento de Estomatología de la Facultad de Odontología de la Universidad de Granada). Agradecimientos especiales a Dña. Gertrudis Gómez Villaescusa (Téc. Laboratorio),

\section{BIBLIOGRAFÍA}

1. Baratieri, LN. Odontologia Restauradora: Fundamentos e Possibilidades. São Paulo. Quintessence. 2001. 1a edição. 83-128.
2. Carvalho RM, Santiago SL, Fernandes CAO, Suh BI, Pashley DH. Effects of Prism Orientation on Tensile Strength of Enamel. J Adhesive Dent 2000; 2: 251-7.

3. Davis WL. Histología y Embriología Bucal. Interamericana. McGraw- Hill.1983. México.

4. Hannig M, Reinhardt KJ, Bott B. Self-Etching Primer vs Phosphoric Acid: An alternative Concept for Composite-to-Enamel Bonding. Oper Dent 1999; 24: 172-80.

5. Hara AT, Amaral CM, Pimenta LAF, Sinhoreti MAC. Shear bond strength of hidrophilic adhesive systems to enamel. Am J Dent 1999; 12: 181-4.

6. Inoue S, Van Meerbeek B, Vargas M, Yoshida Y, Lambrechts P, Vanherle G. Adhesion mechanism of self-etching adhesives. Advanced Adhesive Dentistry 3rd International Kuraray Simposium. 1999.

7. Levinkind M, Vandernoot TJ, Elliot JC. Electrochemical Impedance Characterization of Human and Bovine Enamel. J Dent Res 1990; 69 (12): 1806-11.

8. May KN, Swift EJ, Bayne SC. Bond strength of a new dentin adhesive system. Am J Dent 1997; 10: 195-198.

9. Meurman JH, Frank RM. Progression and surface ultrastructure of in vitro caused Erosive lesions in human and bovine enamel. Caries Res 1991; 25: 81-7.

10. Miyazaki M, Hirohata N, Takagaki K, Onose H, Moore BK. Influence of self-etching primer drying time on enamel bond strength of resin composites. J Dent 1999; 27 (3): 203-7.

11. Mjor IA, Fejerskov. Embriología e Histología Oral Humana. Barcelona. Salvat.1989.

12. Nakabayashi N, Pashley DH. Hybridization of dental hard tissue. Tokyo: Quintessence Publishing Co. 1998.

13. Nakamichi I, Iwatu M, Fusayma T. Bovine teeth as possible substitutes in the adhesion test. $\mathrm{J}$ Dent Res 1983; 62 (10): 1076-81 
14. Oesterle LJ, Shellhart WC, Belanger GK. The use of bovine enamel in bonding studies. Am J Orthod Dentofacial Orthop 1998; 113: 514-9.

15. Reeh ES, Douglas WH, Levine MJ. Lubrication of human and bovine enamel compared in an artificial mouth. Archs oral Biol 1995; 40: 1063-72.

16. Sano H, Ciucchi B, Matthews WG, Pashley DH. Tensile properties of mineralized and demineralized human and bovine dentin. J Dent Res 1994; 73(6).

17. Schwartz RS, Summit JB, Robbins JW. Operative Dentistry: A Contemporary Approach. Illinois. Quintessence Publishing Co. 1996
18. Swift EJ, Perdigão J, Heymann HO, Ritter AV. Shear Bond Strengths of One-Bottle Adhesives to Moist Enamel. J Esthet Dent 1999; 11: 103-7.

19. Sydney-Zax M, Mayer I, Deutsch D. Carbonate Content Developing Human and Bovine Enamel. J Dent Res 1991; 70 (5): 913-916.12.

20. Tay FR, Pashley DH. Aggressiveness of contemporary self-etching adhesives.II. Etching effects on unground enamel. Dent Mater 2001; 17:430-44.

21. Toledano M, Osorio R, De Leonardi G, Rosales JI, Ceballos L, Cabrerizo M. Influence of selfetching primer on the resin adhesion to enamel and dentin. Am J Dent 2001; 14: 205-10. 